\title{
A técnica de análise de micro expressões faciais (METT II-Short) na avaliação da qualidade de jogos digitais: um estudo preliminar de viabilidade.
}

\author{
A case study with the micro expression training tool (METT II-Short). The viability of \\ use in the game quality assurance.
}

\author{
Cláudio Santos Almeida
}

análise, método, técnica, jogos, design, emoção

\begin{abstract}
Este trabalho apresenta um estudo de caso do uso da técnica de análise de micro expressões faciais (METT II-Short) para a coleta de informação a respeito da resposta emocional de jogadores durante uma partida de jogo digital. O objetivo é fornecer subsídios para analisar a viabilidade de uso do METT como instrumento de coleta de $\mathrm{HCl}$. O resultado indica que há a possibilidade de obter resposta acerca do estado emocional do jogador durante uma partida, e que é proveniente do ato lúdico. Essa resposta favorece a um estudo posterior de viabilidade de uso de análises emocionais na análise da qualidade de jogos e a possibilidade de desenvolver um método de âmbito reativo para análises de $\mathrm{HCl}$.
\end{abstract}

analisys, method, technique, games, emotional design

This paper presents a case study with the Micro Expression Training Tool (METT II - Short). The purpose is to identify if this technique is useful to obtain information about players emotional behavior. The objective is to establish the METT viability to $\mathrm{HCl}$ purposes. The results indicates that the technique is useful to obtain information about players emotional behavior. This is a step toward the direction to make a emotional design method for $\mathrm{HCl}$ purposes.

\section{Introdução}

Este artigo apresenta um estudo preliminar da técnica de análise de micro-expressões faciais (METT II Short Version). A técnica foi utilizada na observação de respostas emocionais durante o ato de uso de um jogo digital pelo pesquisador. O objetivo foi o de observar se o instrumental da técnica de análise de micro-expressões faciais METT II Short seria eficaz na coleta e análise de respostas emocionais a estímulos oriundos de uma partida de jogo digital. Para isso, o autor submeteu-se a ser filmado apropriadamente, durante 30 minutos de uma partida de jogo digital monousuário. Essa observação, gravada através de uma câmera a 30 quadros por segundo, foi depois submetida ao teste de análise de micro-expressões faciais contido na ferramenta METT II Short. O objetivo foi encontrar micro-expressões faciais involuntárias que identificasse de forma indelével reações emocionais causadas pelo jogo. O resultado foi positivo, a despeito que serem ressaltadas algumas restrições quanto ao equipamento usado. A confirmação do registro das emoções através de expressões involuntárias foi um passo necessário no intuito de sugerir este instrumento de coleta como ferramenta para auxílio na avaliação de qualidade de jogos digitais. Para que o instrumento possa ser usado efetivamente como ferramenta de avaliação de qualidade, entretanto, outros testes precisam ser realizados.

Este artigo está ordenado da seguinte forma: uma breve descrição da adequação de uso de técnicas de análise emocional em design e $\mathrm{HCl}$; apresentação da técnica de micro-expressões faciais; o estudo de caso realizado; conclusões e desdobramentos.

\section{A análise emocional e o Design da Informação}

O campo teórico do design estabeleceu princípios para o desenvolvimento e sistematização de interfaces de programas há cerca de 30 anos. Os estudos em ergonomia, em usabilidade e em análise de interfaces forneceram as bases para os fundamentos da Relação Humano-Computador $(\mathrm{HCl})$ em design.

Os estudos em HCl centraram-se prioritariamente em aspectos operacionais. Foram desenvolvidos procedimentos para a melhoria dos aspectos operacionais, e cujos fundamentos 
provém dos estudos da usabilidade. Quanto mais fácil de usar, quanto menores os passos e processos necessários à aquisição de conhecimento e proficiência na tarefa, mais eficiente considera-se o software ou sistema.

De acordo com essa abordagem, foram criados métodos necessários à avaliação, análise e aperfeiçoamento dos aspectos que favorecem aos atos de uso dos sistemas por seus usuários. A atribuição de dar aos usuários de sistemas de informação um proveito maior dos recursos informatizados é uma tarefa complexa. Há um vasto conjunto de diferentes tipos de interfaces de softwares. Por conseguinte, foram definidas técnicas de avaliação e análise adequadas. $O$ principal meio epistemológico para a definição dessas técnicas se deu originariamente no âmbito do design e da ciência da computação. Em design, a Ergonomia, o Design de Usabilidade e o Design da Informação são algumas das áreas concernentes. A análise e o aperfeiçoamento das interfaces depende de uma avaliação coerente. Basicamente, (e em larga escala) as técnicas de avaliação de interfaces consistem na observação de sujeitos em relação ao procedimento de determinadas tarefas, em ambientes controlados. O conjunto de variáveis dependentes relacionase com o método aplicado e os resultados são tabulados através de descrições de atividades e/ou de resultados estatísticos.

Inicialmente, em virtude da natureza histórica do desenvolvimento de sistemas de softwares, essas técnicas se adequaram ao requisito básico de modificar processos de interação e favorecer a usabilidade de sistemas. Ao passo, entretanto que os softwares tornaram-se mais complexos e amplos, outras técnicas foram incorporadas ao corpo de conhecimento de $\mathrm{HCl}$ e do design da informação. O objetivo desse aprimoramento passara a ser lidar com um conjunto complexo de variáveis provindo das relações humano-computador. Em particular, com relação às variáveis independentes, como por exemplo, público-alvo, tempo de uso, distância do usuário à interface, seqüência de tarefas realizadas, estas se tornaram muito variantes e de certa forma, tornaram pouco evidente através dos métodos empregados, uma correlação direta com os acontecimentos em ambientes não controlados. As técnicas de coleta e análise dos dados não mais forneceram dados indeléveis compatíveis com resultados práticos de melhoria sistemática, e os fundamentos dos métodos em HCl começaram a mudar. Ainda em meados dos anos de 1980, elementos de tratamento psicológico das informações foram adicionados às técnicas de coleta e análise de $\mathrm{HCl}$, tornando-o uma área interdisciplinar entre design, psicologia e informática. Esse instrumental psicológico facilita a coleta e tratamento de dados gerados por sistemas mais complexos. Suas técnicas de coleta buscam ser confortáveis para os sujeitos da pesquisa e o foco da observação deixa de ser a interface propriamente dita e passa a ser as atividades realizadas pelos sujeitos enquanto usam as interfaces. Essa abordagem, provinda dos estudos psicológicos da cognição criou a análise dialógica de interfaces. Um ambiente dialógico é aquele no qual a interação ou relação entre os elementos de um sistema (seja ele humano-humano, humano-computador ou qualquer outro) é substancialmente um diálogo constante entre as partes. Os métodos de $\mathrm{HCl}$ passaram então a incorporar princípios das teorias que fundamentam o meio dialógico em direção a um instrumental prático, ou seja, a novas técnicas. Um conjunto importante dessas novas técnicas proveio da Análise do Discurso, área preliminar de estudo do campo da Lingüística. $\mathrm{Na}$ figura 1, vemos um modelo clássico de coleta de dados em $\mathrm{HCl}$, utilizando as técnicas de coleta de dados baseado nos questionários escritos e orais (Figura 1).

Figura 1. Modelo clássico de instrumentos de coleta de dados em $\mathrm{HCl}$.

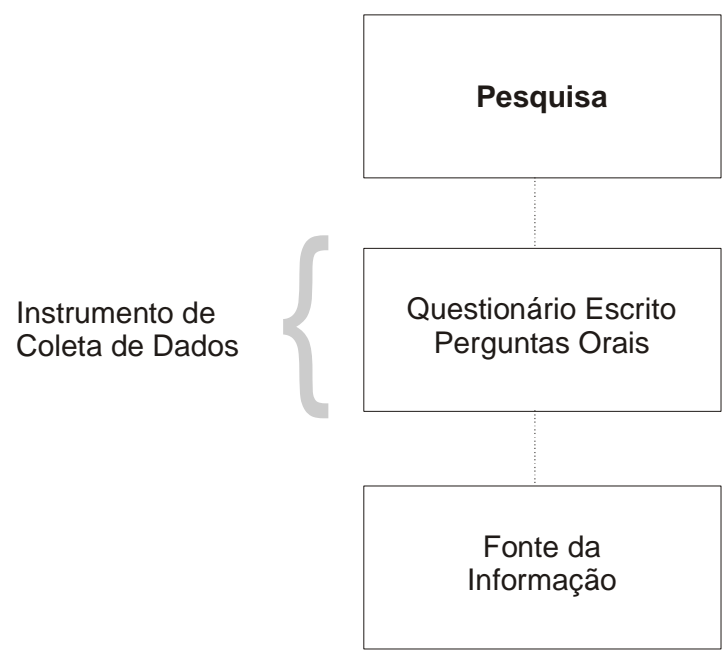


Os modelos baseados no instrumental psicológico e lingüístico oferecem diferentes métodos e técnicas para a coleta dos dados. Abaixo (Figura 2), mostramos um modelo baseado na coleta de dados através da filmagem dos participantes. Esse modelo prescinde de questionários ou perguntas a entrevistados, mas induz os sujeitos a conversarem durante os atos de uso dos objetos de análise. Nesse modelo os dados coletados são, sobretudo, o diálogo existente entre os sujeitos da pesquisa.

Figura 2. Modelo de instrumentos de coleta de dados em $\mathrm{HCl}$ baseado em Análise do Discurso.

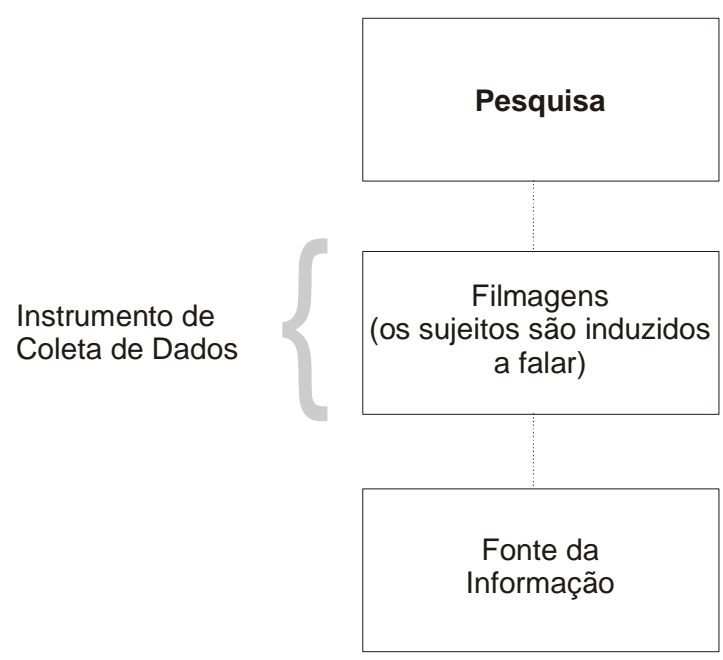

Ambos os instrumentos de análise baseiam-se na coleta de dados de uma resposta oral ou escrita dos sujeitos entrevistados da pesquisa. Esse tipo de coleta serve ao estudo da usabilidade de um largo conjunto de softwares.

O tipo de dado aferido pela análise do discurso difere prontamente do tipo de dados obtidos através de questionários especializados, e dessa forma, as técnicas se complementam.

\section{Técnicas de análise e coleta de dados de resultados de uso de jogos digitais}

Jogos digitais são peças de software e também estão submetidos aos princípios que regem a relação homem-computador e, portanto, interfaces. Por exemplo, assim como em qualquer software, em jogos digitais também há uma curva de aprendizagem, um sistema de entrada e saída e assim por diante. Em jogos digitais, entretanto, em virtude da natureza lúdica do ato de jogar, o intuito de utilizar o sistema pode ser simplesmente a busca por satisfação, cujo fundamento é prioritariamente proveniente de respostas emocionais. Não pertence ao escopo desse artigo a descrição pormenorizada de princípios da natureza do jogo e do ato lúdico. Uma descrição mais detalhada pode ser encontrada em Huizinga, nos aspectos formativos e filosóficos e Kishimoto nos aspectos técnicos e contextuais. (Huizinga, 2004; Kishimoto, 1998).

Por conta de aspectos concernentes ao jogo, a dinâmica do uso de jogos digitais difere consideravelmente da dinâmica de uso de outros tipos de softwares, e deve-se levar em consideração que, assim como em qualquer jogo, digital ou não, o jogo é a atividade fim. Ou seja, diferente de quando se usa um software especialista, não se espera produzir nada com o jogo além do próprio ato de jogar.

Quando se trata de jogos digitais o uso de instrumentos de coleta que evoquem ou necessitem de respostas verbais ou escritas - seja através de questionários, seja através da indução da fala na linguagem oral, parece não ser o mais apropriado. Há vários aspectos que indicam isso, sendo que um dos principais consiste na natureza particular do ato lúdico - que ocorre durante uma partida de jogo digital, e que consiste de um isolamento do mundo exterior ao jogo. Outro aspecto importante é que há questões pertinentes à forma como ocorre a disposição dos sujeitos no uso de jogos digitais individualmente, ou ainda quando os sujeitos jogam jogos digitais em rede. Cada jogador, de uma forma ou de outra, se concentra normalmente no seu jogo e a existência de duplas não é a regra. Não é possível, a mais das vezes, falar em um diálogo direto, e não é 
recomendado interromper uma partida com o objetivo de coletar dados. Uma ação desse tipo pode interferir fortemente no ato lúdico e modificar os resultados dos dados obtidos.

Essa questão, que diz respeito aos atuais métodos e técnicas de coleta de dados disponíveis em HCl, quando utilizados para a avaliação e análise de jogos digitais, situa-se exatamente na correlação entre a natureza da coleta, o tipo de dado coletado e a forma como esses dados devem ser analisados para comporem um resultado eficiente no aprimoramento dos artefatos. Essa característica tem relação com o tipo de resultado esperado de um jogo digital e de um software. Diferentemente das atribuições de um software operacional, por exemplo, um editor de texto, não é suficiente para um jogo ser fácil de ser jogado, ter uma curva de aprendizagem suave ou ter elementos gráficos que atendam prontamente a conceitos ergonômicos de usabilidade. Um jogo, diferentemente de um software operacional, não tem apenas uma função de eficiência. Tem também uma função lúdica - de entretenimento.

Qualquer método e técnica empregada para a avaliação, análise e aprimoramento de jogos digitais e deve levar em consideração o aspecto lúdico, além de ser eficiente ter sob si o cabedal teórico que justifique epistemologicamente sua validade.

\section{A análise de micro expressões faciais como possível instrumento de coleta de dados em jogos digitais.}

Micro Expression Training Tool (METT) é Estado da Arte em técnicas de análise facial nos Estados Unidos da América e foi lançado para o uso de pesquisadores em junho de 2008. METT existe atualmente em três versões. Diferem quanto ao tempo de treinamento e o tipo de especificidade a que se destinam. O que se observa, através do uso da técnica é um tipo de reação muscular involuntária que ocorre em seres humanos, de difícil contenção e que tem sua origem em reações emocionais. Através de estudos sistemáticos por mais de quarenta anos, os pesquisadores desenvolvedores das ferramentas de análise de micro-expressão facial chegaram à conclusão que é possível encontrar sete tipos de micro expressões universais, ou seja, cujo significado emocional é o mesmo em todas as culturas e etnias estudadas. A cada uma dessas micro-expressões universais está associado um único estado emocional (Figura 3).

Figura 3. Exemplo de captura de micro-expressão. A expressão facial dura tipicamente menos que um segundo.

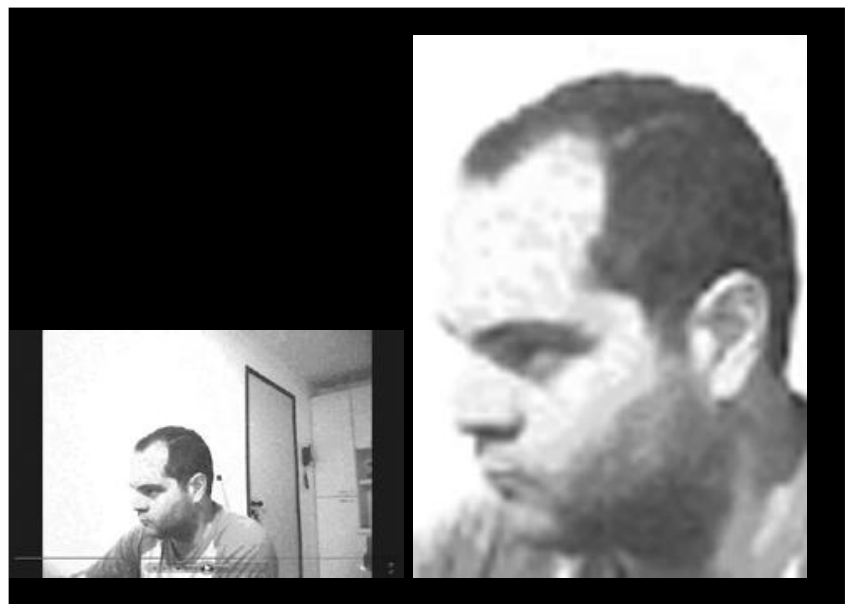

As reações faciais são chamadas de micro-expressões por conta do seu tempo de duração, que tipicamente dura em torno de 1/15 de segundo. Em virtude da rapidez com que ocorrem, não são percebidas habitualmente.

A análise das expressões faciais é um dos instrumentos usados para análise emocional na psicologia emocional. Seu fundamento é explicado sucintamente a seguir:

A investigação de resultados de ações corporais provindos de respostas a estados emocionais remonta a Charles Darwin. Em seu livro "The Expression of Emotions in Man and Animals", Darwin faz observações acerca da expressão corporal e da expressão facial em seres humanos. A questão que Darwin lançou dizia respeito aos grupos musculares, em particular da face, que são difíceis de serem estimulados conscientemente. Ele questionou se, por serem difíceis de serem acionados voluntariamente, também seria difícil suprimir seu movimento em ações involuntárias. Por exemplo, se o grupo de músculos responsáveis pelo sorriso verdadeiro, em particular aqueles 
que se movem involuntariamente, também se moveriam a despeito de tentar se evitar a apresentação de um sorriso involuntário. Se isso fosse verdade, então seria possível identificar emoções através do rosto, mesmo quando a reação facial a elas associada fosse suprimida. Ekman estendeu esses estudos. Ele realizou experimentos que evidenciaram a existência de pequenas reações musculares faciais, a que denominou de micro-expressões. Essas microexpressões seriam respostas a estímulos emocionais suprimidos, pelo menos parcialmente, por uma atitude consciente. Em seus estudos, Ekman estava interessado em obter informação acerca da dissimulação e da mentira. Sua intenção foi a de verificar se seria possível averiguar a mentira contada por sujeitos através das ações involuntárias de sua musculatura facial. Seu resultado foi, na ocasião, de cerca de $80 \%$ de acerto, o que validou a análise de micro-expressões como instrumento de avaliação de respostas emocionais associadas a ações. (Ekman, 1993; Ekman, 1999; Ekman, 2004; Damasio, 1996).

Informação mais acurada sobre o METT, bem como sobre a base teórica do estudo das microexpressões pode ser encontrado em http://www.paulekman.com.

O objetivo da pesquisa foi verificar se a técnica funciona para observar emoções durante $o$ ato de jogar um jogo digital. Idealmente, se essa condição provasse ser verdadeira, então uma proposição como a que é exposta abaixo seria possível (Figura 4):

Figura 4. A análise de expressões faciais como instrumento de coleta de dados de resposta emocional no uso de games.

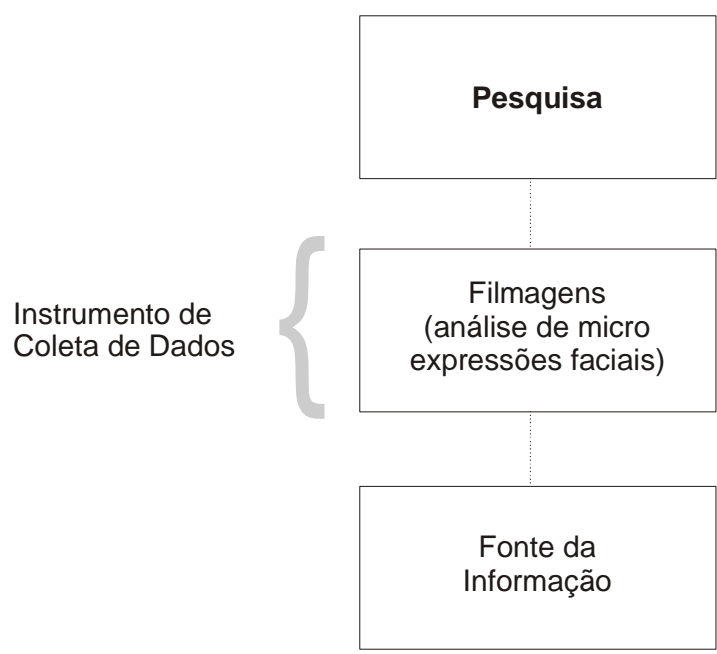

A metodologia a seguir foi utilizada com o objetivo de oferecer subsídios para o uso de METT como instrumento de coleta de dados em $\mathrm{HCl}$, particularmente em sistemas lúdicos, como jogos digitais. A vantagem dessa técnica é que ela é essencialmente discreta. Nenhuma pergunta precisa ser feita e o pesquisador sequer precisa estar presente. Uma câmera filmadora é usada para filmar os participantes. A câmera deve possuir certo conjunto mínimo de atributos. Dessa filmagem podem ser extraídos os dados da pesquisa.

\section{0 experimento.}

A pesquisa foi realizada pelo autor como sujeito de uma partida de jogo digital. $O$ jogo escolhido foi Warcraft III, extensão Frozen Throne (Figura 5). O jogo Warcraft III é um Real Time Strategy Game, em 3D da fábrica de jogos Blizzard. Warcraft é um dos temas mais vendidos de jogos para Pcs e seu universo temático agrega mais de cinco milhões de usuários. A escolha do jogo segue os seguintes critérios: em primeiro lugar, é um jogo conhecido do autor. Em segundo, teoricamente por se tratar de um jogo com forte narrativa e campanhas determinadas, haveria uma menor possibilidade de surpresas durante $o$ ato lúdico. O que se chama campanhas determinadas consiste na rigidez linear de ação. No jogo individual de Warcraft III, não há caminhos, ou paths diferentes sendo os mesmos em cada partida. O objetivo foi criar dificuldades para testar o METT como instrumento de coleta. Quanto mais adversas fossem as condições, mas consistente seria a comprovação da adequação da ferramenta ao objetivo pretendido. 
Figura 5.Warcraft III. Um dos mais vendidos títulos de jogos digitais. Narrativa convincente associada a um cuidado gráfico acurado fizeram do tema um sucesso de vendas.

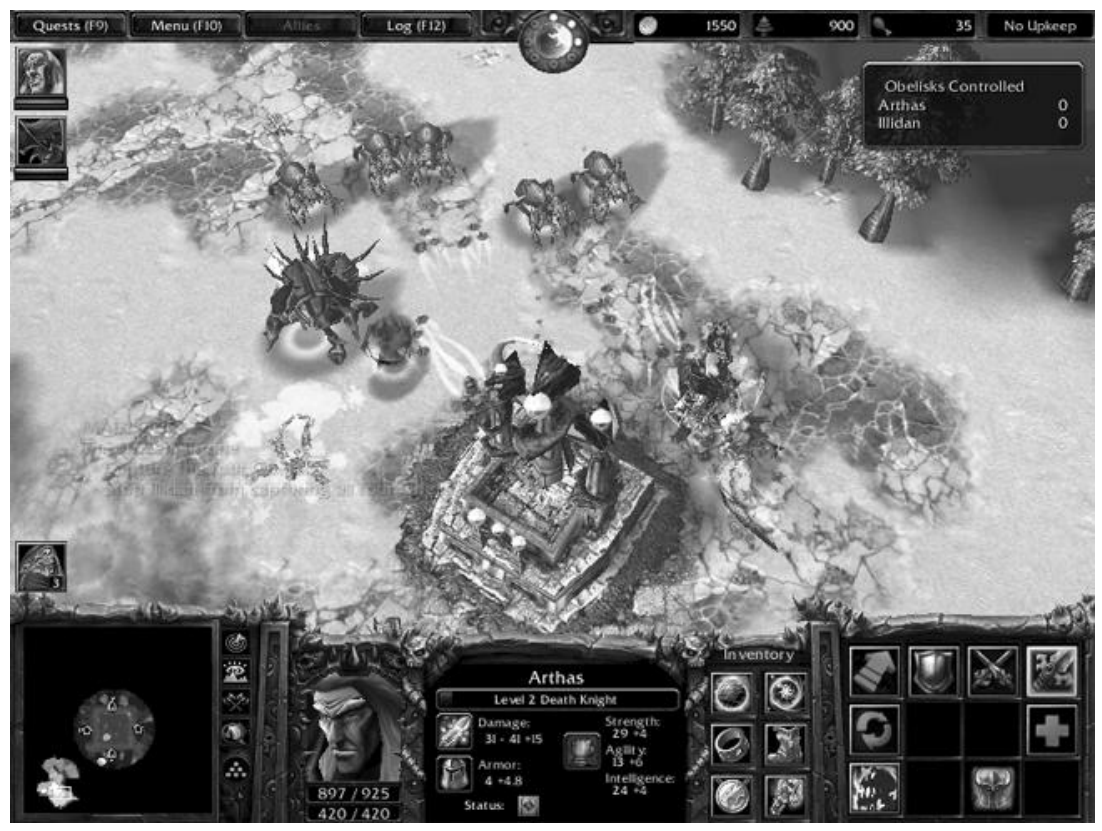

\section{Equipamento}

A captura foi realizada com uma webcam embutida, do tipo Crystal Eye Webcam, em um notebook Acer 5520-5912. A placa de vídeo usada para a captura é uma Nvídia 7000 séries. A taxa de quadros por segundo ficou em torno de 20 (20 Fps). A resolução de saída foi $640 \times 480$.

O jogo Warcraft III foi jogado com teclado e mouse em um Desktop Core 2 Duo, com 2 GB de memória RAM e uma placa de vídeo GE Force 8500 GT, com 512 MB, o que permite jogar com máxima qualidade gráfica e performance adequada.

\section{Filmagem}

Realizou-se a filmagem com o notebook colocado lateralmente, fazendo a webcam um ângulo de aproximadamente 45 graus com o sujeito (Figura 6).

Figura 6. Posicionamento para a filmagem.

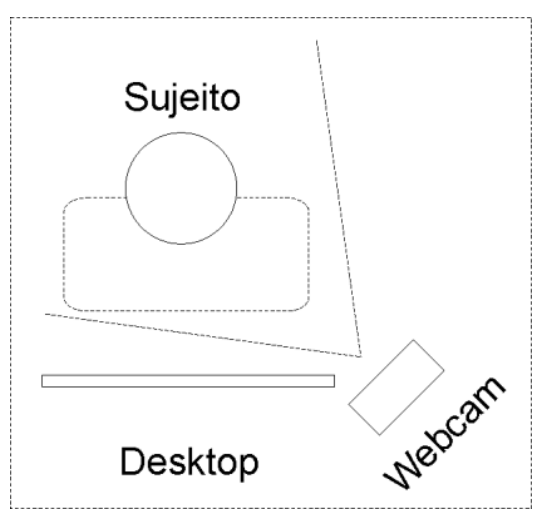

Foi utilizado o software Debut Vídeo Capture Software, versão 1.34, que permite demarcar o tempo de filmagem, após o que o software automaticamente encerra a filmagem e grava um arquivo de vídeo. O tempo determinado foi de 30 minutos. Esse tempo foi demarcado levando-se em consideração dois aspectos: é um tempo razoável de filmagem para uma partida de Warcraft III, que pode durar algumas horas. Desta forma, consideramos que um tempo mínimo de 30 minutos seria aceitável para promover o envolvimento com o jogo. O segundo aspecto foi o estudo 
do tamanho de arquivo, uma vez que gravamos com um formato de compressão simples. A estimativa foi de que o arquivo tivesse algumas centenas de megabytes para trinta minutos de gravação, o que foi comprovado.

\section{Avaliação}

Depois de concluída a filmagem, procedeu-se duas análises das imagens do vídeo a partir dos preceitos da técnica de análise de micro-expressões. A condição mais favorável seria a da redução da velocidade de reprodução do vídeo a cerca da metade. A qualidade da filmagem, porém, que não chegou a $30 \mathrm{Fps}$ não permitiu essa abordagem. Foi feita, então uma série de investigações em partes da filmagem. Essa abordagem permitiu encontrar várias situações em que ocorreram claramente micro-expressões faciais. Particularmente entre o minuto 14 e 15 até o minuto 20 as micro-expressões claramente denotam uma relação emocional com a partida. A avaliação, portanto, quanto à possibilidade de uso foi positiva.

A filmagem lateral prejudicou a análise e a baixa taxa de quadros por segundo também. Indicações para que sejam feitas filmagens frontais com uma taxa maior de quadros é bastante relevante.

$\mathrm{O}$ arquivo gerado ficou com algo em torno de $1 \mathrm{~GB}$ de tamanho, o que evoca para filmagens maiores um bom sistema de armazenamento, tal como um HD externo ou fitas DAT. Um DVD fornece autonomia para cerca de duas horas de gravação nessas taxas.

Figura 7. Exemplos de captura de micro expressões. Observe-se a variação de expressão nos dois últimos exemplos, a $18 \mathrm{~m} 42 \mathrm{~s}$ e $19 \mathrm{~m} 25 \mathrm{~s}$. As micro-expressões não duram um segundo completo e são de difícil visualização a olho nu.

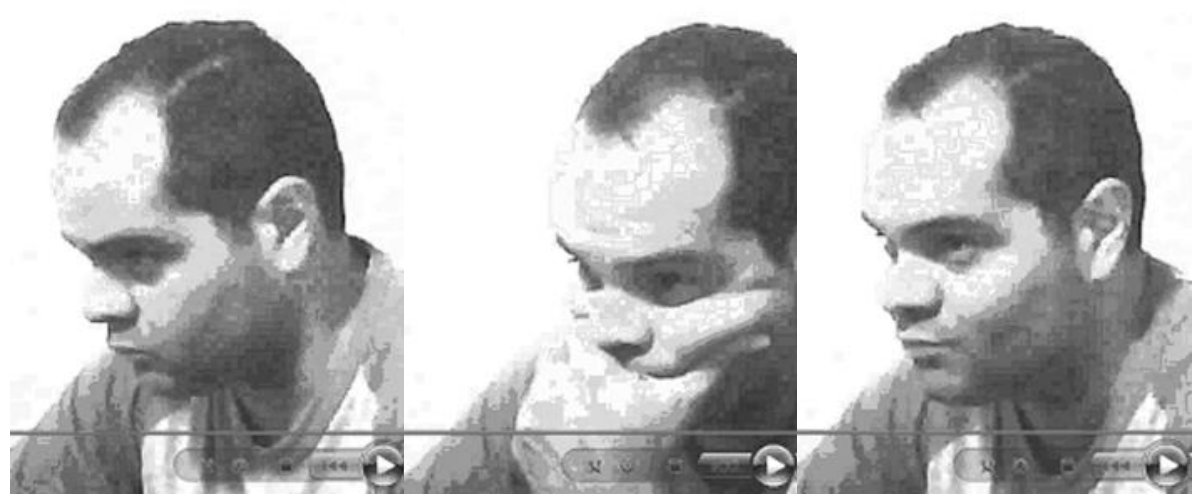

\section{Conclusão e desdobramentos.}

A primeira abordagem de uso do instrumental de METT foi bem sucedida quanto à sua possibilidade de uso como instrumento de coleta de dados de reações emocionais durante o uso de jogos digitais.

É interessante notar que o sujeito da pesquisa estava consciente dos objetivos da filmagem e da técnica de micro-expressões, e devido a isso procurou evitar a ocorrência espontânea de expressões faciais. Não obstante, estas ocorreram. De fato, as micro-expressões são aparentemente involuntárias, o que é condizente com os estudos de Ekman (Ekman, 1993).

Um aspecto a ser ressaltado é que embora tenha sido possível gravar micro-expressões, seria também possível que essa gravação em nada tivesse a haver com o jogo. Em outras palavras, seria possível gravar micro-expressões em qualquer atividade. Justamente por isso, a recíproca é verdadeira - seria possível então gravar também micro-expressões associadas aos atos do jogo. Para clarificar esse ponto, procurou-se por representações de micro-expressões que não tivessem ligação com os eventos externos ao jogo. Procurou-se por emoções que devessem estar ligadas prioritariamente às sensações emocionais do jogo.

Embora durante os quinze primeiros minutos da gravação, não houvesse nada que indicasse claramente que as micro-expressões estavam relacionadas com a partida jogada, perto de vinte 
minutos, a quantidade e o tipo de seqüência das micro-expressões denotou claramente uma relação com os acontecimentos da partida. Procuramos evitar filmar o jogo, uma vez que a percepção dos acontecimentos da partida poderia induzir o pesquisador a procurar microexpressões em intervalos específicos, e dessa forma conduzir a pesquisa. Não houve, portanto, informação direta sobre os acontecimentos do jogo e sim sobre o que aconteceu emocionalmente com o jogador.

A técnica das micro-expressões permite identificar sete tipos de diferentes emoções, que são independentes de cultura. Esse não foi, entretanto, o objetivo da pesquisa. Para esse objetivo específico foi preparado outra coleta, com três sujeitos, em circunstâncias semelhantes de uso de um console de jogo. Nesse caso, pretende-se verificar além da ocorrência das micro-expressões, o tipo de emoção associado. O objetivo é montar futuramente uma matriz de reações emocionais. Essa matriz pode vi a permitir identificar padrões emocionais subjacentes que correspondam a uma avaliação reflexiva do usuário.

\section{Referências.}

Damásio, Antônio R. 1996. O erro de Descartes emoção razão e o cérebro humano. Tradução de Dora Vicente e Georgina Segurado. 1.ed. São Paulo: Companhia das Letras, 324p.

Ekman, P. 1999. Basic Emotions. In: T. Dalgleish and M. Power (Eds.). Handbook of Cognition and Emotion. Sussex, U.K.: John Wiley \& Sons, Ltd.

Ekman, P. 1993. Facial Expression and Emotion. In: American Psychologist, v. 48, n. 4, p. 384-392.

Ekman, P.; Campos, J.; Davidson R.J.; De Waals. 2003. F. Darwin, Deception, and Facial Expression. In: Emotions Inside Out. Volume 1000. New York: Annals of the New York Academy of Sciences, p. 205-221.

Huizinga, Johan. 2004. Homo Ludens. 5.ed. São Paulo: Perspectiva, 243p.

International Council Of Societies Of Industrial Design. Disponível Em: <http://www.icsid.org/about/about/articles31.htm?query_page=1>. Acesso em: 14 Mai. 2008.

Itiro, lida; Mühlenberg, Poema. 2006. O Bom E O Bonito Em Design. In: Congresso Brasileiro de Pesquisa e Desenvolvimento em Design, 7., Paraná. Anais...Paraná: P\&D.

Kashimura, K.; Kurosu, M. 1994. The Structure Of The Screen Design And The Cognitive Process. In: Japanese Psychological Association, 58., Disponível em:

<http://www.sigchi.org/chi95/proceedings/shortppr/mk_bdy.htm>. Acesso em: 15 Mai. 2008.

Kishimoto, Tizuko Morchida. 1998. O Jogo E A Educação Infantil. 1.Ed. São Paulo: Pioneira, 63p.

Norman, Donald A. 2005. Emotional Design: Why We Love (Or Hate) Everyday Things. [1th] Ed. New York: Basic Books, 257p

Ono, Maristela Mitsuko. 2006. Design E Cultura: Sintonia Essencial. 1.Ed. Curitiba: Edição da Autora, 132p.

Padovani, Stephania. 2002. Avaliação Ergonômica de Sistemas de Navegação em Hipertextos Fechados. In: Moraes, A. (Org.). Design e Avaliação de Interface. 1. Ed. Rio De Janeiro: Luser, p. 30-31.

Petterson, Rune. 2007. Research in Information Design. In: Congresso Internacional de Design da Informação, 3., Curitiba. Anais...Curitiba: SBDI.

Definição de Design da Informação. In: Sociedade Brasileira de Design da Informação. Disponível em : <http://www.sbdi.org.br/>. Acesso em: 04 abr. 2009.

Spinillo, Carla; Farias, Priscilla. 2007. Design da informação em mídias digitais interativas. In: Infodesign. Revista Brasileira de Design da Informação. n.2, v.4. Disponível em: <http://www.infodesign.org.br/>. Sessão: <Outros números><No. 2 | Vol. $4-2007><$ Editorial>. Acesso em: 04 abr. 2009.

Tractinsky, Noam. 1997. Aesthetics and Apparent Usability: Empirically Assessing Cultural and Methodological Issues. Disponível em: http://www.sigchi.org/chi97/proceedings/paper/nt.htm. 